\title{
La Gouvernance, entre innovation et impuissance
}

Le cas de l'environnement

Jacques Theys

\section{(2) OpenEdition}

Journals

Édition électronique

URL : http://journals.openedition.org/developpementdurable/1523

DOI : 10.4000/developpementdurable.1523

ISSN : $1772-9971$

Éditeur

Association DD\&T

Référence électronique

Jacques Theys, "La Gouvernance, entre innovation et impuissance », Développement durable et territoires [En ligne], Dossier 2 | 2003, mis en ligne le 01 novembre 2003, consulté le 30 avril 2019.

URL : http://journals.openedition.org/developpementdurable/1523; DOI : 10.4000/

developpementdurable.1523

Ce document a été généré automatiquement le 30 avril 2019

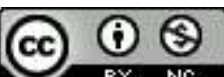

Développement Durable et Territoires est mis à disposition selon les termes de la licence Creative Commons Attribution - Pas d'Utilisation Commerciale 4.0 International. 


\title{
La Gouvernance, entre innovation et impuissance
}

\author{
Le cas de l'environnement
}

Jacques Theys

1 Le terme de "gouvernance » s'est imposé au cours des années 90 comme le symbole d'une nouvelle modernité dans les modes d'action publique et le gouvernement des entreprises ; et est devenu, en quelques années, un des lieux communs du vocabulaire de l'aménagement du territoire. Si récemment l'affaire ENRON a pu contribuer à en démystifier les promesses, les critiques - même justifiées - qu'a pu susciter ce concept ${ }^{1}$ ne doivent pas faire oublier la part incontestable d'innovation démocratique qu'il recèle. Le domaine de l'environnement, qui a joué sur ce thème un rôle précurseur, est sans doute celui où l'on mesure le mieux cette ambiguité. Et c'est ce qui justifie qu'il soit pris ici comme exemple - d'autant que les points communs avec l'aménagement du territoire sont, comme on le constatera, multiples.

2 L'environnement a été en effet depuis plusieurs décennies, et reste aujourd'hui encore, un "laboratoire" où s'inventent en permanence de nouvelles formes de gouvernance: procédures démocratiques de consultation, formes flexibles de coordination, modes de gestion décentralisés, utilisation du contrat, de la médiation ou des incitations économiques, gouvernement par l'information et les principes... On pourrait multiplier les exemples qui témoignent de cet effort de modernisation de l'action publique: "Agendas 21 ", "études d'impact" , "accords volontaires" , "marchés de droits à polluer”, " autorités indépendantes" , “conférences de consensus", " principe de précaution", "développement durable" , "normes ou conventions négociées"... Constatons simplement qu'à l'évidence cette tendance s'est notablement accélérée au tournant des années 90 - dans un contexte marqué par la globalisation, l'émergence des grandes régions économiques comme l'Europe, et l'influence croissantes des idées 
libérales - au point que l'on peut parler rétrospectivement d 'une véritable "révolution silencieuse " dans la conception et la mise en œuvre des politiques de l'environnement.

Cet essor tout particulier des nouvelles formes de gouvernance dans le champ de l'environnement s'explique aisément par au moins trois raisons convergentes :

- d'abord par la nature même des problèmes concernés : problèmes d'externalité, de gestion de risques ou d'utilisation des ressources communes, allant du niveau local au niveau planétaire. Par définition, ces problèmes sont complexes, conflictuels, controversés, et leur solution passe par la mobilisation d'acteurs nombreux, interagissant à de multiples échelles ; des acteurs dont les territoires ne correspondent généralement pas aux territoires institutionnels classiques, et qu'il faut convaincre ;

- par ailleurs l'environnement est lui-même porteur de valeurs favorables à la démocratie, à la décentralisation, à la transparence et donc à des formes d'action publique qui accordent une large place à la société civile ;

- il est clair, enfin, que les nouvelles formes de gouvernance ont aussi été pour les politiques de l'environnement, un moyen de surmonter leurs faiblesses ou leur déficit de légitimité, et en particulier de réagir aux critiques d'inefficacité ou d'autoritarisme auquel elles ont été confrontées dès l'origine.

4 Même si l'environnement joue ainsi un rôle " d'avant garde" dans la modernisation des formes de gouvernance, il ne faut pas, cependant, oublier que cette volonté d'innovation coexiste avec le souci quasi obsessionnel de renforcer les modes d'intervention traditionnels de l'Etat - de type "command and control". On est encore en effet dans une phase où il s'agit prioritairement pour les responsables de l'environnement, de fonder et construire une politique sectorielle bien identifiée, avec des moyens d'autorité classique (réglementation, dispositifs de contrôle, ressources budgétaires), et des instruments de coercition suffisants pour obtenir des résultats tangibles à courte échéance.

5 Autrement dit, la nécessité d'innover coexiste avec la volonté de trouver une place légitime parmi les politiques traditionnelles de l'Etat - et donc de se couler dans le " moule" des politiques classiques d'interventions, de réglementation et de sanction.

6 Cette situation de coexistence historique entre deux priorités différentes, entre deux stratégies d'action publique, alimente naturellement beaucoup de controverses. D'un côté, on accuse les politiques réglementaires traditionnelles d'être archaïques, inefficaces, inapplicables. De l'autre, on considère que les nouvelles formes de gouvernance ne sont qu'une façon naïve d'évacuer la réalité des conflits et des jeux de puissance, et que sous couvert d'ouverture démocratique elles ne font que renforcer les intérêts dominants et institutionnaliser les corporatismes. Au-delà, ce sont deux conceptions de la démocratie qui s'affrontent avec un défi commun : comment organiser la confrontation des opinions et des intérêts sur des questions essentiellement médiatisées par la science, qui transcendent les frontières institutionnelles, et concernent des générations ou des éléments de la nature qui n'ont pas accès au vote?

7 En conséquence, le débat sur la "gouvernance » est généralement enfermé dans deux discours contradictoires. Pour les uns la “ bonne gouvernance " est la seule solution adaptée aux problèmes actuels d'environnement, et elle a donc vocation à se substituer aux politiques publiques traditionnelles considérées comme dépassées. Pour les autres, au contraire, la « gouvernance » est le problème - et non la solution - car elle ne fait que renforcer l'impuissance collective face à des défis de plus en plus ingouvernables. En 1995, William D. SUNDERLIN, dans un article portant sur le 
changement global ${ }^{2}$, mettait déjà en évidence l'éclatement des réflexions des chercheurs autour des trois catégories "paradigmatiques": d'un coté, ceux qui ont une vision essentiellement managériale de la "gouvernance »; de l'autre, ceux qui insistent sur les évolutions ou les différenciations culturelles; et enfin, ceux qui ont une vision "agonistique", en terme de "rapports de force", du problème, et considèrent que les solutions passent nécessairement par des changements structurels improbables (gouvernement mondial, leadership européen...).

Cette dichotomie conduit à une impasse. Plutôt que d'opposer une vision idyllique de la «bonne gouvernance» à un pessimisme radical, il est urgent de construire des passerelles entre ces différentes positions; et de se placer non pas dans une logique de substitution des formes démocratiques de gouvernance aux modes classiques d'action publiques - mais plutôt dans une perspective de complémentarité et de nouvelle “ division de travail " entre l'Etat et la société civile, entre la démocratie représentative et la démocratie délibérative, entre l'innovation managériale et la crédibilité institutionnelle. Cela revient à mettre la gouvernance à l'épreuve des réalités, ou si l'on préfère à l'épreuve de la "gouvernabilité" (et de ses contradictions). Cela conduit aussi, finalement, à poser la question qui semble centrale dans tous ces débats sur la gouvernance, et rejoint la perspective culturaliste évoquée par Sunderlin: à quelles conditions, peut-on aujourd'hui construire en Europe et ailleurs une démocratie cognitive qui permette de donner une réalité concrète à la participation du public dans les décisions?

1. Gouvernance, gouvernabilité ou gouvernementalité?

9 Même si le terme de "gouvernance" fait aujourd'hui partie d'un patrimoine international commun ; même si - comme on vient de le dire - les gestionnaires de l'environnement en font désormais un usage courant, il n'en reste pas moins un concept extrêmement vague, très marqué par la culture anglo-saxonne - et donc exposé à des interprétations nécessairement diverses et subjectives. Cela justifie, avant d'aborder plus concrètement le thème de l'environnement, que l'on s'arrête un moment sur les problèmes de définition, ne serait-ce que pour expliciter les présupposés idéologiques qui accompagnent souvent l'usage - ou l'abus d'usage - de ce mot.

10 Ce détour, nécessaire, par les dictionnaires (et les définitions) restera ici très sommaire. Il ne s'agira naturellement pas de proposer un cadre théorique sur la gouvernance participative $^{3}$, ni même d'ouvrir la controverse sur le différents sens du mot "gouvernance ", mais seulement de choisir un angle de vue ou une perspective utile pour éclairer le sujet de "la gouvernance environnementale " abordé ici.

11 Pour des raisons qui apparaîtront plus clairement ultérieurement, il semble bien qu'une des façons les plus commodes, mais sans doute aussi parmi les plus efficaces, d'éclairer ce qui fait l'originalité du concept de gouvernance, c'est de le comparer à d'autre termes assez voisins comme ceux de : "Gouvernabilité" ou de "Gouvernementalité ".

12 Les similitudes, les complémentarités, mais aussi les oppositions et les contrastes entre chacun de ces trois termes sont en effet un bon révélateur de ce qui fait la richesse sémantique du concept de "gouvernance" - mais aussi de ce qui en constitue les limites évidentes en particulier dans ses rapports avec la démocratie.

1.1. La Gouvernance : boîte à outils de l'interaction non hiérarchique

13 Que nous disent les dictionnaires à propos de la gouvernance ? En fait, peu d'indications précises. Pour le WEBSTER'S NEW UNIVERSAL DICTIONNARY, par exemple, il s'agit à la 
fois de " la forme du régime politique "; “"du processus par lequel l'autorité est exercée dans la gestion des ressources économiques ou sociales"; ou "de la capacité des gouvernements à concevoir, formuler et mettre en œuvre des politiques et, en général à assumer leurs fonctions gouvernementales ${ }^{5 "}$.

Prises en tant que telles, toutes ces définitions restent, comme on le constate, extrêmement vagues, et renvoient à une partie très traditionnelle de la science politique. Elles n'expliquent pas, à l'évidence, pourquoi l'utilisation de ce terme de gouvernance s'est développée depuis une dizaine d'années dans une perspective beaucoup plus spécifique, et sans doute aussi, beaucoup plus normative.

Cette explication, il faut, naturellement, la trouver dans le contexte même des années 80 : dans la montée de la globalisation et des flux transnationaux; dans la révolution de l'information et des technologies de la communication; dans la perte croissante de légitimité des Etats et d'efficacité de régulation politique ; dans la fin de la bipolarisation Est-Ouest... Et ce n'est pas un hasard si, par exemple, la revue "Gouvernance " naît à peu près en même temps que la chute du mur de Berlin. Si l'on parle de "gouvernance " c'est en effet que cette "gouvernance" est en crise; et que l'on pense que cette crise, qui est essentiellement une crise de l'action publique substantialiste et des "mandats d'autorité" pourra être dépassée par de meilleures formes d'organisation, de gouvernement ou de management - la Banque Mondiale parle ainsi, dès 1989, de "Good governance".

on utilise le mot "gouvernance", on ne se réfère donc certainement pas aux définitions très larges données par le WEBSTER'S Dictionnary mais plus probablement à cette définition beaucoup plus explicite, malgré les apparences, donnée par la Commission BRANDT sur la "Gouvernance globale", et reprise, ensuite, par Eric BAIL au nom de la Commission Européenne : "La somme des voies et moyens à travers lesquels les individus et les institutions, publiques ou privées, gèrent leurs affaires communes. Il s'agit d'un processus continu grâce auquel les divers intérêts en conflit peuvent être arbitrés et une action coopérative menée à bien. Ceci inclut les institutions formelles et les régimes chargés de mettre en application les décisions, ainsi que les arrangements que les gens ou les institutions ont acceptés ou perçoivent comme étant dans leur intérêt ${ }^{6}$ ".

17 L'important dans cette définition c'est l'idée de processus interactif: une succession d'étapes à travers lesquelles des acteurs nombreux n'ayant pas le même intérêt et agissant à différentes échelles, mais confrontés à un même problème vont progressivement construire une représentation commune de cette réalité, lui donner un sens, se fixer des objectifs, adopter des solutions puis les mettre en œuvre collectivement sans que rien - ni cette représentation, ni ces objectifs, ni ce sens, ni cette interaction ne soient déterminés à l'avance. ${ }^{7}$

Essentiellement pragmatique, le concept de "gouvernance " renvoie ainsi finalement à une "boîte à outils" - une liste très impressionnante et extensible de recettes managériales ou d'instruments supposés apporter des réponses appropriées à la crise des politiques démocratiques traditionnelles, centrées sur l'autorité de l'Etat (cf. le tableau 5). L'histoire à succès de ce mot - qui comme on l'a dit ne peut se comprendre qu'en référence au contexte actuel - s'explique sans doute ainsi par le sentiment - on pourrait presque parler de croyance mythique - que nous avons maintenant à notre disposition un "livre de cuisine politique moderne", comprenant toutes les recettes nécessaires et suffisantes pour permettre de surmonter les contradictions de l'action collective. Un catalogue d'outils "universels" capables de répondre à toutes les situations, même les 
plus complexes, sans aucune vision idéologique du "bon gouvernement" - si ce n'est une vision plutôt abstraite de la démocratie, conçue comme une interaction ouverte et pluraliste entre acteurs.

19 C'est cette vision neutre, optimiste, managériale de l'action collective qui est mise fortement en doute par les deux autres termes de "gouvernabilité" et de "gouvernementalité".

1. 2. La Gouvernabilité : réaffirmation de la singularité des situations et critique des solutions universelles.

20 Si la notion de "gouvernance " concerne plutôt les outils et les processus de l'action collective, celle de "gouvernabilité » met l'accent sur la spécificité des situations, et sur la probabilité, face à ces situations spécifiques, plus ou moins complexes, de trouver des solutions à la fois efficaces et acceptables. Certaines situations sont intrinsèquement ou politiquement gérables; d'autres ne le sont pas - ou ne peuvent l'être qu'au prix de difficultés et d'efforts considérables. Ce qui veut dire que les solutions vont fortement dépendre de la nature des problèmes ou de la structure des relations existantes entre les différents acteurs.

21 Vont ainsi intervenir de manière déterminante : le caractère plus ou moins structuré ou controversé des questions mises en jeu, l'existence ou pas de solutions praticables, le nombre des acteurs impliqués dans cette solution et leur capacité à se coordonner, l'accord ou pas sur les objectifs et les moyens d'action, la capacité à gouverner du ou des principales instances de coordination ou des acteurs majeurs (ressources, savoir faire, légitimité, organisation), et enfin la volonté ou l'habileté de ces acteurs majeurs à élaborer un projet de gouvernement crédible et susceptible de générer des alliances stables et suffisamment puissantes.

22 Concrètement la notion de gouvernabilité débouche ainsi sur les typologies de situations plus ou moins "gouvernables » et à toute une série de distinctions entre, par exemple, problèmes bien structurés et mal structurés; modes de gouvernement en univers stabilisé ou en univers controversés ${ }^{8}$; gouvernabilité forte, moyenne ou faible ${ }^{9}$.

23 Ce qui est important, par rapport au thème de la gouvernance démocratique, c'est que la notion de gouvernabilité remet finalement en cause l'idée même "d'outil universel », de boîte à outils appropriée à toute les situations. Dans certains cas de «basse gouvernabilité » (problèmes non structurés ou sans solution praticable, pluralité anarchique d'acteurs opposés, capacité de blocage d'un acteur majeur, faiblesse structurelle des institutions, absence de projet mobilisateur...) les gains à attendre de procédures plus ouvertes de "gouvernance » seront mineurs. Et puis surtout les « styles de gouvernement ", et donc les processus à mettre en place, seront très différents selon la nature des problèmes en jeu et les contextes institutionnels. C'est ce qu'Olivier GODARD a remarquablement formalisé dans le domaine de l'environnement en opposant de manière radicale la prise de décision en "univers stabilisé" et en "univers controversé" (tableaux 1 et 2).

24 C'est aussi, l'idée défendue de manière convaincante par Yves Mény et Jean-Claude Thoenig ${ }^{10}$, qui différencient fortement plusieurs types possibles de processus ou styles de gouvernement plus ou moins efficaces - en fonction du degré d'accord ou de désaccord entre acteurs sur les objectifs et les valeurs (première dimension), et du degré de certitude ou d'incertitude existant sur le fait et les moyens à mettre en œuvre (seconde dimension). Dans certaines situations les contradictions pourront être gérées par la 
négociation ou le pragmatisme; dans d'autres cas on n'évitera pas un processus chaotique essentiellement régulé par les crises (tableau 3).

Tableau I et II : Modes de gouvernement dans deux univers de décision différents (stabilisé et controversé) (source Olivier Godard)

\section{Tableau I : La prise de décision en univers stabilisé}

\begin{tabular}{|c|}
\hline $\begin{array}{l}\text { Les agents ont une perception directe des effets externes ou des biens } \\
\text { colectifs } \\
\text { Leurs preferences sont bien intormees }\end{array}$ \\
\hline $\begin{array}{l}\text { Seuls les interèts ou preférences des agents presents sont directement } \\
\text { pertinents }\end{array}$ \\
\hline $\begin{array}{l}\text { Ces agents disposent de procedures sociales adequates pour exprimer } \\
\text { leurs preterences : } \\
\text { marche, votes, manifestations et protestations, contits }\end{array}$ \\
\hline $\begin{array}{l}\text { La connaissance scientifique s'est stabilisee sur les aspects des } \\
\text { problemes pertinents pour laction : } \\
\text { - chaines causales élucidees } \\
\text { - dommages bien constituees } \\
\text { - imputation des responsabilites denuees dambiguité }\end{array}$ \\
\hline $\begin{array}{l}\text { Les phenomenes en cause sont reversibles : } \\
\text { on peut attendre un developpement suifisant des connaissances pour } \\
\text { pouvoir prendre des décisions contorme aux exigences du modele de } \\
\text { la rationalite substantielle (analyses coots - avantages) }\end{array}$ \\
\hline $\begin{array}{l}\text { Les connaissance scientifiques stabilisees constituent un monde } \\
\text { oommun pour tous les acteurs, de façon prealable a laction }\end{array}$ \\
\hline $\begin{array}{l}\text { L'enjeu de la situation : lefficacite economique et lequite, sur la base } \\
\text { d'interéts bien constitués }\end{array}$ \\
\hline
\end{tabular}

\section{Tableau II : La prise de décision en univers controversé}

Prédominance de la construction scientifique et sociale des problemes sur la perception directe par les agents

La représentation separée des interéts de tiers absents est en cause : génerations futures, Butres pays, especes naturelles, biosphere (?) lis sont des porte-parole contradictoires

La connaissance scientifique est encore controversée sur des aspects essentiels du probleme pertinents pour l'action

Du fait de lirreversibilite potentielle, et du caractere majeur des enjeux, certains acteurs estiment qu'li faut aqir immediatement. sans attendre la. stabilisation des connaissances

Les théries scientifiques, les "visions du monde et du futur"

deviennent des variables strategiques donnant naissance a de

nouvelles tormes de compétition

L'enjeu de cette compétition:

la formation de communautes epistemiques et la fixation de

conventions denvironnement 
Tableau III Nature des problèmes et processus ou styles de gouvernement (Source : Y. Mény et J. C. Thoenig)

\begin{tabular}{|c|c|c|c|}
\hline & & \multicolumn{2}{|c|}{$\begin{array}{c}\text { Degré d'accord sur } \\
\text { les objectifs } \\
\text { et les valeurs }\end{array}$} \\
\hline & & ELEVE & FAIBLE \\
\hline \multirow{4}{*}{$\begin{array}{l}\text { Degre de } \\
\text { certutude sur } \\
\text { les moyens, } \\
\text { les taits, la } \\
\text { connaissance }\end{array}$} & \multirow[b]{2}{*}{$\begin{array}{l}\text { ELE } \\
\text { VE }\end{array}$} & $\begin{array}{l}\text { Processus } \\
\text { programme }\end{array}$ & $\begin{array}{c}\text { Processus } \\
\text { négoclé }\end{array}$ \\
\hline & & $\begin{array}{c}\text { Routines: } \\
\text { automaticites: } \\
\text { non-venement. } \\
\text { Technicisation, } \\
\text { bureaucratisation, } \\
\text { planification. }\end{array}$ & $\begin{array}{c}\text { Débats } \\
\text { ideologiques: } \\
\text { recours a } \\
\text { Pexperience et.a } \\
\text { la tradition. } \\
\text { Controverse } \\
\text { officielles et } \\
\text { compromis } \\
\text { informels. }\end{array}$ \\
\hline & \multirow{2}{*}{$\underset{E}{\text { FAIBL }}$} & $\begin{array}{l}\text { Processus } \\
\text { pragmatique }\end{array}$ & $\begin{array}{l}\text { Processus } \\
\text { chaotique }\end{array}$ \\
\hline & & $\begin{array}{l}\text { Recours aux } \\
\text { experts", } \\
\text { empirisme } \\
\text { (le mieux qu'on } \\
\text { peut) recherche } \\
\text { de vanantes } \\
\text { strategiques }\end{array}$ & $\begin{array}{l}\text { Evitement: } \\
\text { decentralisation, } \\
\text { recours a lautorite } \\
\text { ou a fhomme } \\
\text { providentiel; } \\
\text { gestion de crises. }\end{array}$ \\
\hline
\end{tabular}

Tout cela, naturellement, est bien connu des spécialistes de sciences politiques. Mais l'immense mérite du concept de gouvernabilité est finalement de nous rappeler en permanence que les processus de gouvernement ne sont pas dissociables des contextes structurels dans lesquels ils se déroulent, ce que l'idée de "boîte à outils universelle » conduit parfois à faire oublier.

1.3. La «Gouvernementalité » : Une critique de la neutralité idéologique du "bon gouvernement".

26 Malgré leurs différences, les deux précédents concept de "gouvernance " et de « gouvernabilité » ont néanmoins en commun d'être très forcément liés à "l'analyse de système ». Ils s'inspirent clairement d'une conception managériale des systèmes politiques pour laquelle il s'agit essentiellement de trouver des solutions pragmatiques à des défaillances de marché ou à des défaillances d'intervention publique. Mais peut-on parler de «bonne gouvernance» - ou de "gouvernabilité forte ou faible" - sans vision normative du bon gouvernement?

27 Cette question est naturellement controversée: à la perspective fonctionnaliste, «cybernétique », et apparemment apolitique de la «gouvernance » défendue et symbolisée par des personnalités majeures de la science politique américaine comme Karl Deutsch ou David Easton, s'oppose une vision plus historique et idéologique de la "gouvernementalité ", dont le meilleur représentant est sans doute le philosophe français Michel Foucault - auquel on doit ce troisième et dernier terme.

Pour Karl Deutsch, qui a joué un rôle majeur dans la filiation entre cybernétique et gouvernance ${ }^{11}$, celle-ci s'apparente en effet essentiellement à un ensemble fonctionnel d'instruments de contrôle et de guidage. Un ensemble de régulations qui permettent au système politique de s'adapter à son environnement, et font intervenir de manière centrale la capacité à diffuser, échanger, recevoir de l'information. Comme l'indique le titre même d'un de ses livres majeurs: The Nerves of Government ${ }^{12}$, ce qui compte dans le 
système politique, ce ne sont pas "les muscles ou les os" (c'est à dire les «rapports de force ») mais «les nerfs»: «il est donc plus profitable - écrit dès $1963 \mathrm{~K}$. Deutsch d'étudier le gouvernement non pas comme un phénomène illustrant la présence du pouvoir, mais comme un instrument de guidage, le guidage étant avant tout une question de communication. S'il y a dysfonctionnement du système politique, c'est parce qu'il n'est plus capable de déchiffrer ou capter les informations essentielles, ou parce qu'il y a un écart croissant entre l'interdépendance des acteurs et l'échange d'information ». Conçue ainsi comme un ensemble de mécanismes autorégulateurs, d'incitations et de signaux, la gouvernance ne suppose, dans cette perspective, aucune vision politique ou éthique du bon gouvernement, aucune conception du monde ou "méta-rationnalité », si ce n'est un vague assentiment sur des règles purement procédurales et cognitives de transparence, de réflexivité et d'accès à l'information. Elle n'intègre pas non plus la dimension du temps et de l'irréversibilité, qui est pourtant, comme on le sait, essentielle pour déterminer l'efficacité des mécanismes « d'autorégulation » mis en jeu par la communication.

La position de Michel Foucault est, dans une large mesure, symétrique de celle de Karl Deutsch: là où ce dernier privilégie l'autorégulation sans rupture, la neutralité du fonctionnalisme, et les vertus de la rationalité communicatrice, il réintroduit la discontinuité historique, la spécificité du pouvoir, et l'importance des visées politiques. Pour lui, nous ne pouvons comprendre les pratiques pragmatiques, les arrangements collectifs, le fonctionnement des outils ou des procédures en les isolant des objectifs et valeurs assignées à l'action publique ; mais surtout en oubliant les formes de rationalité ce qu'il appelle lui-même "les régimes de vérité » - qui structurent en profondeur ces pratiques ou ces arrangements. C'est cette combinaison des outils, des objectifs et des systèmes de rationalité qui définit la "Gouvernementalité $»^{13}$ - un art de gouverner dont les formes changeantes sont indissociablement liées à l'histoire: de même que Machiavel introduit une rupture radicale par rapport au modèle politique de la Renaissance; de même ne peut-on assimiler l'art de gouverner orienté par la «Raison d'Etat » à celui qui se construit à partir du $18^{\text {ème }}$ siècle, autour de la conception libérale de la politique (tableau 4), puis du « biopouvoir ». 
Tableau IV : Raison d'état et libéralisme : deux formes de gouvernementalité (Source : Michel Foucault)

\begin{tabular}{|c|c|c|}
\hline $\begin{array}{r}\text { Formes de } \\
\text { gouvernement }\end{array}$ & RAISON D'ETAT & LIBERALISME \\
\hline $\begin{array}{l}\text { Objectif du } \\
\text { gouvernement }\end{array}$ & $\begin{array}{l}\text { - Construire un état } \\
\text { souverain et pu'ssant }\end{array}$ & $\begin{array}{l}\text { - Accroltre la } \\
\text { prospente et la liberte } \\
\text { individuelle }\end{array}$ \\
\hline Question Majeure & - Y a till assez $d$ etat & $\begin{array}{l}\text { - Comment gouverner } \\
\text { a la limite du trop ou } \\
\text { trop peu de } \\
\text { gouvernement? }\end{array}$ \\
\hline $\begin{array}{l}\text { Instruments de } \\
\text { politique interne }\end{array}$ & $\begin{array}{l}\text { - Pouvair de police } \\
\text { limite }\end{array}$ & $\begin{array}{l}\text { - Autolimitation du } \\
\text { pouvoir de police } \\
\text { - Incitations }\end{array}$ \\
\hline $\begin{array}{l}\text { Régulation et } \\
\text { objectifs externes }\end{array}$ & $\begin{array}{l}\text { - Souveraineté et } \\
\text { autonomie des Etats } \\
\text { - Concurrence } \\
\text { - Pouvoir limité a } \\
\text { l'extérieur }\end{array}$ & $\begin{array}{l}\text { - Commerce } \\
\text { - Cosmopolitisme } \\
\text { - Interdependance }\end{array}$ \\
\hline $\begin{array}{l}\text { Sources de } \\
\text { limitations du } \\
\text { pouvoir }\end{array}$ & $\begin{array}{l}\text { - Limite externe } \\
\text { (culture) }\end{array}$ & $\begin{array}{l}\text { - Limite interne } \\
\text { (nature) }\end{array}$ \\
\hline $\begin{array}{l}\text { Formes rationnelles } \\
\text { de limitation et de } \\
\text { réguiation du } \\
\text { pouvoir }\end{array}$ & $\begin{array}{l}\text { - Droit } \\
\text { - Legitimite (fondation } \\
\text { du pouvoir) }\end{array}$ & $\begin{array}{l}\text { Economie politique } \\
\text { - Efficacite \{effet de } \\
\text { l'action\} }\end{array}$ \\
\hline
\end{tabular}

La bonne gouvernance ne serait-elle alors qu'une version modernisée - adaptée à la complexité et à la globalisation - du modèle libéral du gouvernement tel que décrit par Michel Foucault ${ }^{14}$ ? On peut faire l'hypothèse - mais naturellement tout ceci est discutable - qu'il s'agit plutôt de l'amorce d'un nouvel "art de gouverner", original combinant sans doute une forte proportion d'individualisme et de libéralisme, mais avec au moins trois ou quatre des "ingrédients" suivants :

- la confiance dans l'auto-organisation de l'action collective ;

- l'intégration de formes plus réflexives ou procédurales de rationalité ${ }^{15}$;

- le développement de valeurs plus "cosmopolites" à l'échelle de la planète ;

- l'adhésion croissante aux idées formalisées par Ulrich BECK, François EWALD ou Hans JONAS autour de la "Société du risque" et du "Principe de précaution" ;

- Une conception plus ouverte et participative de la démocratie « as a mechanism by which as many people as possible have optimal influence on the political conditions which affect their lives, relations, work and environment »;

- et enfin très probablement, une certaine "dose" de "despotisme éclairé" - à travers le pouvoir croissant des experts, ce qui n'est pas sans contradiction avec l'idéal démocratique précédent...

Naturellement on pourrait aussi considérer, comme l'a fait par exemple Francis Fukuyama ${ }^{16}$, que l'émergence de la gouvernance - comme ensemble universel de solutions fonctionnelles - clôt définitivement la question posée par Michel Foucault du sens politique et historique des formes de gouvernement. Qu'elle marque, d'une certaine façon la "fin du politique"17.

Mais l'expérience de l'environnement, qui va maintenant être abordée, est là pour nous montrer les limites d'une vision purement apologétique de la «bonne gouvernance » même participative - qui serait débarrassée des contextes réels dans lesquels elle se 
déploie ("la gouvernabilité"), et des raisons politiques qui lui donnent un sens ("la gouvernementalité"). Autrement dit, on ne peut s'en tenir à la simple description de la boîte à outils: il faut aussi essayer d'expliquer pourquoi elle est ou pas utilisée; et pourquoi, dans certains cas, elle ne fonctionne pas.

1.4. La gouvernance démocratique comme solution aux nouveaux enjeux de l'environnementa) Le tournant des années 90

Toutes les réticences que l'on peut raisonnablement avoir vis à vis d'un discours beaucoup trop "irénique" sur la gouvernance n'enlèvent rien à la réalité du constat qui introduisait ce papier : les politiques de l'environnement ont été, surtout à partir des années 90, un remarquable laboratoire pour des formes nouvelles de gouvernance. Et celles-ci ont incontestablement constitué des avancées significatives dans le fonctionnement démocratique partout où elles ont été développées - en ouvrant à un nombre croissant d'acteurs l'opportunité d'intervenir dans la conception et la mise en œuvre de solutions collectives à des problèmes de mieux en mieux perçus comme communs.

Il n'est donc pas totalement surprenant que certains auteurs comme Anthony Giddens ou Ulrich Beck soient allés jusqu'à dire que «la crise environnementale avait joué un rôle central ("a seminal role") dans l'émergence de la "modernité réflexive" - comme d'ailleurs dans la modernisation de la vie politique »: c'est sans doute dans ce domaine que l'expérimentation de nouvelles formes d'action collective a été la plus vive.

Au tournant des années 90 , il devînt en effet évident que tout progrès supplémentaire dans la politique de l'environnement dépendait désormais de la capacité à mobiliser la société toute entière - en commençant par les entreprises, les consommateurs et les habitants. "L’intégration", "l'internalisation", "la responsabilisation", "la participation" devinrent de nouveaux slogans largement répandus. Au même moment le processus conjoint de globalisation, de décentralisation et de constitution de grandes "régions économiques" (CEE, ALENA...) conduisit progressivement à un encadrement sensible des Etats-Nations, contraints, désormais, de négocier ou de s'ajuster avec une pluralité diffuse d'acteurs influents intervenant à toutes les échelles - du global au local. Dans un contexte d'incertitude scientifique et de complexité croissante, un consensus très large se forma sur la nécessité de faire évoluer la politiques de l'environnement du curatif au préventif (puis à la précaution); de l'injonction à la participation; de la centralisation à la décentralisation; de l'orientation par l'Etat à une orientation par le marché ; de l'excès de réglementation à une action essentiellement incitative, créant les conditions favorables a un changement dans les comportements des producteurs ou des consommateurs...

b) Une gouvernance à quatre dimensions

36 Prises ensemble, toutes ces transformations de l'action publique dessinent aujourd'hui un "paysage" apparemment très sophistiqué de la "bonne gouvernance"; un enchevêtrement très complexe de principes, d'instruments, d'institutions et de procédures dont on discerne mal, au premier abord, la cohérence.

37 En réalité, au-delà de cette apparente confusion, on constate assez aisément que ce concept commun de "gouvernance environnementale" recouvre quatre approches ou quatre réalités assez différentes (tableau 5):

- un souci évident de relégitimation et de modernisation de l'action publique - qui passe d'abord par plus de transparence ; 
- des formes originales et multiples de coordination non hiérarchiques (et de tranversalisation) des actions collectives ;

- le passage à des formes plus ouvertes de rationalité (réflexive, procédurale...) ;

- et enfin, il ne faut pas l'oublier, un certain transfert de pouvoirs vers la société civile, les collectivités décentralisées ou des institutions autonomes (nationales ou internationales).

- La première approche est très classique. Il s'agit essentiellement de rationaliser, crédibiliser, moderniser l'action publique : rendre les administrations "comptables" de leur action ( «accountability ), donner plus d'indépendance à l'expertise (création d'autorités indépendantes...), réduire la bureaucratie, développer la transparence et l'accès à l'information, favoriser la participation aux décisions... L'objectif - qui est de reconstruire une certaine confiance envers les institutions - n'est naturellement pas spécifique à l'environnement : mais c'est, à l'évidence un domaine où les problèmes de crédibilité, de transparence et de légitimité se posent avec une acuité particulière, compte tenu de l'importance des conflits et des incertitudes qui s'y manifestent.

- Moderniser l'action publique ne suffit cependant pas : dans une seconde approche de la "bonne gouvernance", c'est plus fondamentalement le principe même de mandat d'autorité ou d'autorité hiérarchique qui est remis en cause - à la fois pour des raisons d'efficacité mais aussi du rôle de fait joué par une multiplicité d'institutions ou d'acteurs à toutes les échelles. L'extraordinaire éclatement des enjeux et des pouvoirs impliqués dans les problématiques écologiques suppose des mécanismes de coordination non hiérarchiques de plus en plus sophistiqués - procédures de négociation, système de partenariat et de contrats, conventions internationales, instruments de médiation, mécanismes de marché (marché de droit à polluer...), accords de subsidiarité... qui sont pour beaucoup de praticiens, au centre de la notion de gouvernance.

- Quelle que soit l'importance de ces questions de coordination, de "couturage" des institutions et des acteurs, c'est sans doute dans une troisième approche qu'il faut chercher ce qui fait véritablement l'originalité de la gouvernance environnementale. De manière encore plus ambitieuse que la précédente cette troisième approche vise, finalement, à élargir les représentations traditionnelles de la rationalité ; à dépasser les cadres trop étroits de la rationalité instrumentale à court terme. Concrètement cela s'est traduit, en particulier dans la période récente, par une remarquable capacité à "inventer" - puis à diffuser - de nouveaux principes d'action : "principe pollueur payeur", "principe de précaution", "développement durable" ... . Mais aussi par une extension considérable du champ de la réflexivité dans la prise de décision (études d'impact, calcul économique, outils de "reporting", évaluation des risques, etc.). L'intérêt de ces principes est naturellement qu'ils peuvent s'accommoder de formes très souples de relations entre acteurs, ou même d'absence de relations (dans la perspective qui est celle de "l'individualisme méthodologique").

- Ce serait pourtant manquer l'essentiel que de réduire la "bonne gouvernance" à cet effort très ambitieux pour fonder sur des bases rationnelles plus larges une nouvelle action collective. L'essentiel en effet, dans la "bonne gouvernance", c'est une redistribution des pouvoirs et des rôles entre l'Etat, les autres institutions locales ou internationales, le marché et la société civile. D'un mode de gouvernement où l'Etat, centralisant les responsabilités, déterminait seul l'action des autres acteurs, on passe à un mode de gouvernement où tous les acteurs concernés exercent collectivement cette responsabilité. 
Tableau 5 : Les quatre dimensions de la gouvernance environnementale

\begin{tabular}{|c|c|}
\hline $\begin{array}{l}\text { 1- Modemiser l'action publique, } \\
\text { en accroitre la légitimite et la } \\
\text { crédibilité } \\
\text { (Gerer la conflance } \\
\text { et I'acceptabilité) }\end{array}$ & $\begin{array}{l}\text { 2- Developper des mecanismes } \\
\text { non autoritaires de } \\
\text { coordination et de régulation } \\
\text { de faction collective } \\
\text { (Gerer la pluralite } \\
\text { et la mobilisation) }\end{array}$ \\
\hline $\begin{array}{l}\text { - Reforme du secteur public } \\
\text { - Transparence } \\
\text {-Evaluation, controle, } \\
\text { "accountablity' } \\
\text { - Autorités independantes } \\
\text { - Separation regulateur/ } \\
\text { operateur } \\
\text { - Consultations et debats publics - } \\
\text { democratisations des procedures } \\
\text { - Mise en ceivure plus efficace } \\
\text { (enforcement") }\end{array}$ & $\begin{array}{l}\text { - Extension du contrat } \\
\text { - Partenariat public-prive } \\
\text { - Incitations economiques (permis } \\
\text { negociables, taxes) - } \\
\text { compensations } \\
\text { - Accords volontaires } \\
\text { - Conventions et protocoles } \\
\text { flexibles ("accords cadres") } \\
\text { - Politiques constitutives } \\
\text { - Rutorites regulatrices } \\
\text { - Mecanismes de mediation } \\
\text { - integration et transversalisation } \\
\text { - Mise en oeuvre negociee } \\
\text { - Reseaux intormels }\end{array}$ \\
\hline $\begin{array}{l}\text { 3- Etendre la rationalité réflexive } \\
\text { ou procedurale } \\
\text { (Gérer l'incertitude } \\
\text { et la complexité) } \\
\text { - Principe de precaution } \\
\text { - Developpement durable } \\
\text { - Evaluation des risques, etudes } \\
\text { d'impact. rétiexivité } \\
\text { - Calcul economique et reformes } \\
\text { comptables } \\
\text { - Acces a linformation, } \\
\text { transparence, traçabilite, } \\
\text { indicateurs, audits... } \\
\text { - Conferences de consensus } \\
\text { - Pluralite de l'expertise, autorites } \\
\text { independantes } \\
\text { - Science "post normale* } \\
\text { - Deontologle et comites déthique }\end{array}$ & $\begin{array}{l}\text { 4- Changer de pouvoir } \\
\text { (Gerer les rapports de force) } \\
\text { - Transterts de souveraineté (aux } \\
\text { institutions supranationales) } \\
\text { - Decentralisation } \\
\text { - Subsidiarite active } \\
\text { - Droits de propriete } \\
\text { - Normalisation volontaire } \\
\text { (exigences essentielles, } \\
\text { autocertifiction) } \\
\text { - Delegation au secteur prive ou } \\
\text { aux O.N.G } \\
\text { - Autogestion' des biens publics } \\
\text { par des communautes d'usagers } \\
\text { - Institutions de mutualisation } \\
\text { (agences de reau) }\end{array}$ \\
\hline
\end{tabular}

Cette transition est bien illustrée dans les deux graphiques suivants dus à Pierre-Frédéric Tenieres-Buchot, qui synthétisent, de façon simple, la "révolution" introduite par les Agences de l'Eau en France. A une période (graphique 1) où l'Etat, investi de la responsabilité essentielle en matière de l'eau, cherchait à l'obtenir par l'incitation, la contrainte (" command and control ») et l'investissement direct, succède une seconde phase (graphique 2) où cette responsabilité est désormais assurée par la communauté des usagers. Dans cette nouvelle étape, l'Etat conserve néanmoins deux rôles non négligeables: mettre en place les mécanismes de subsidiarité, et surtout, évaluer les résultats du dispositif, et rendre cette évaluation publique.

Graphiques 1 et 2 : Les transformations de la gouvernance dans le domaine de l'eau en France (Source : P.-F. Tenieres-Buchot) 
Graphique 1 : L'Etat régalien

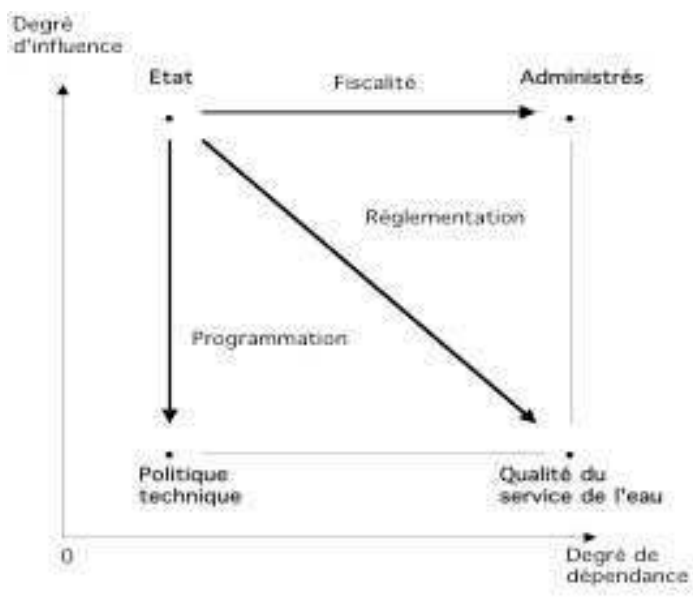

Graphique 2 : Gestion de l'eau par la communauté des usagers

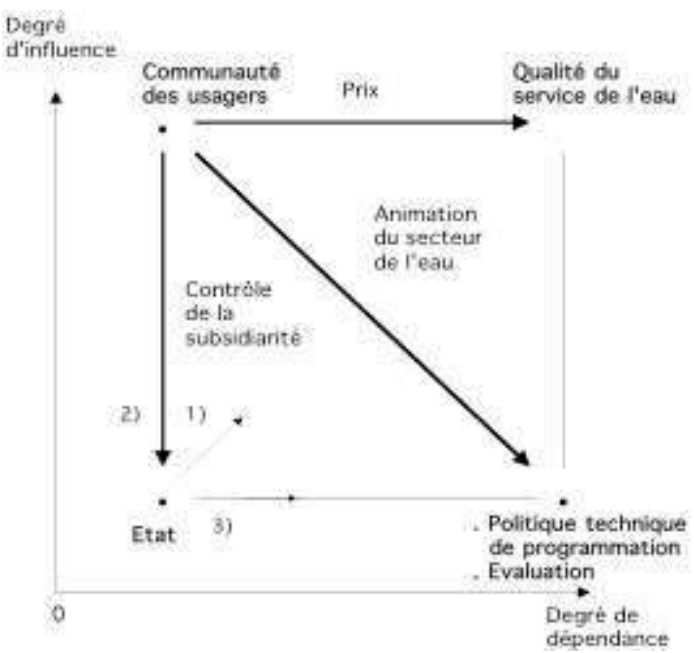

L'objectif, finalement, n'est plus seulement d'introduire plus de rigueur dans l'action administrative (première conception), de trouver de nouveaux modes de coordination (seconde conception) ou d'étendre les critères de la rationalité (troisième conception) ; mais de redistribuer très largement le pouvoir autour de nouvelles formes de solidarité - solidarités du voisinage, solidarités régionales... Ce qui donne à la gouvernance un sens nécessairement politique.

c) Des formes très différentes d'implication démocratique

Ce qui est important quand on considère cette "boîte à outil" de la gouvernance, en expansion continue, ce n'est pas seulement cette coexistence entre quatre approches, quatre dimensions différentes; mais aussi le fait que ces quatre composantes recouvrent des conceptions très contrastées du processus démocratique, et des relations entre les institutions représentatives et la société civile.

- Dans la première perspective, qui est celle de la modernisation de l'action publique, chacun est, en principe, appelé à participer aux décisions qui le concernent - de la manière la plus large. Mais l'objectif majeur de ce processus participatif "ouvert" reste, en définitive, la justification et la relégitimation des institutions (des Etats aux collectivités locales, en passant par la Commission Européenne). L'enjeu demeure le plus souvent celui de l'acceptabilité des 
politiques publiques, de la restauration de la confiance, et du "contrôle" de l'opinion - même si cette volonté de canalisation est en partie contrebalancée par l'exigence croissante de transparence et d' " accountability », par l'extension du syndrome NYMBY... et les incertitudes inhérentes à la médiatisation.

- Dans la seconde perspective - celle d'une meilleure efficacité des mécanismes de coordination - la participation est souvent limitée à un nombre restreint d'institutions intervenant à différentes échelles - et à un cercle relativement réduit de "parties prenantes" ( «stakeholders ») qui ont ainsi l'opportunité de négocier des contrats ou des engagements flexibles - dans un processus de marchandage et d'adaptation mutuelle. L'intérêt des nouvelles formes de gouvernance - du point de vue de l'environnement - est qu'elles rendent possible la représentation, dans ce processus de marchandage, de nouveaux intérêts et en particulier des organisations non gouvernementales, associations écologiques ou de consommateurs... Mais cette ouverture, par ailleurs réelle, du processus de décision ne peut généralement se faire que dans la mesure où ces représentants de la société civile acceptent un langage de négociation communqui reste principalement un langage économique ${ }^{18}$.

- Dans la perspective qui est celle d'un changement de formes de rationalité, et d'une intégration progressive de nouveaux principes d'action, chaque individu ou chaque organisation est placée devant sa propre responsabilité : à chacun de prendre ses propres décisions puis de les mettre en œuvre, dans le cadre d'une "éthique de la responsabilité" commune. Tout le monde, d'une certaine manière est dans la position du responsable politique, et participe à une politique globale décrite par Ulrich BECK comme celle d'une "repolitisation de la sphère privée" ou ce qui revient au même d'une "privatisation de la fonction politique" (ce qu'Ulrich BECK appelle aussi le développement de la "sub-politique"). A un autre niveau, plus idéologique ou discursif ("the meta level") l'accent mis sur les principes ouvre aussi un espace de débat intéressant entre les experts et le public le plus large - par exemple sur les règles d'équité ou de responsabilité vis à vis des générations futures - et ceci ne peut que favoriser la dynamique démocratique.

- Dans la quatrième et dernière dimension de la gouvernance - celle d'un nouveau partage des pouvoirs - les responsabilités sont, par définition décentralisées au profit de la société civile - ou du moins d'une partie d'entre elle. En pratique cette délégation ne bénéficie le plus souvent qu'à un nombre limité d'usagers, de riverains ou de secteurs économiques... intéressés à la gestion d'un même bien commun. On peut en attendre beaucoup plus qu'une simple participation : une réelle redistribution du pouvoir réel de décision. Mais il n'est pas évident que ce processus de délégation de pouvoir soit réellement démocratique : on est en effet le plus souvent dans une logique d'organisation de service où il s'agit de faire participer les usagers à des formes de partenariat public-privé. Et la question de la responsabilité (« accountability ») des ces "communautés d'usagers" ou de ces "co-gestionnaires de biens communs" par rapport au système démocratique légalement élu reste posée - comme cela a été effectivement le cas en France pour les Agences de l'Eau.

41 Si l'on croise les quatre configurations précédentes, nul ne peut douter que les évolutions récentes de la gouvernance environnementale ont considérablement ouvert les opportunités d'implication et de participation d'acteurs qui auparavant étaient exclus ou sous-représentés dans le processus de décision: organisations non gouvernementales, groupes d'intérêt, consommateurs, riverains des infrastructures, collectifs scientifiques, leaders d'opinion... L'intervention croissante de l'Europe, par exemple, et les procédures de consultation qu'elle a su mettre en place, ont donné à des intérêts sectoriels, à des associations, à des régions... des marges de manœuvre dont ils ne disposaient pas au niveau national. Mais cette redistribution des cartes dans le jeu démocratique s'est, en même 
temps, accompagnée d'un autre déplacement, tout aussi important, qui est celui des enjeux politiques de la négociation sur l'environnement. Comme le remarquait en 1996 Jan Van Tatenhove $^{19}$, le passage à ce qu'il appelle "la modernisation politique réflexive" s'est en effet traduit par un recentrage du débat institutionnel et de la problématique écologique autour de nouvelles questions, désormais "dominantes", comme l'intégration dans le marché, les enjeux commerciaux, l'articulation "global" - "local", et, dans un autre registre, la gestion de la précaution et des rapports à la science. Or il n'est pas aujourd'hui évident que ce "recadrage" soit nécessairement favorable à l'implication démocratique du plus grand nombre - ou qu'il débouche sur une prise en compte plus responsable des générations futures. Il pose, en tout cas, un problème majeur qui est celui de l'articulation entre les nouvelles formes de participation de la société civile et les structures de la démocratie représentative - dont la légitimité est simultanément ébranlée.

d) L'idéal d'une société civile mondiale

Paradoxalement la transition vers des processus plus modernes de gouvernance semble, en définitive, plus facile à l'échelle internationale qu'à celle des Etats ou des collectivités locales: non seulement plus de trois cents textes régissent aujourd'hui le droit de l'environnement ; mais surtout on constate que beaucoup de ces textes, par la diversité des principes qu'ils affichent ou l'originalité des dispositifs qu'ils mettent en place, "tirent vers le haut" les politiques nationales et jouent, à l'évidence, un rôle moteur dans la modernisation de l'action collective.

Il y a à cela plusieurs bonnes raisons : la souplesse des "régimes internationaux" - et sans doute une moindre rigidité des arrangements institutionnels mis en jeu; une culture internationale plus ouverte au pragmatisme et au consensus; ou encore la possibilité, au niveau régional ou global, de "jeux à somme positive" (win - win) - liés aux conséquences inattendues des interdépendances entre pays. Mais il ne faut pas négliger une autre raison, sans doute moins avouable: le sentiment général que ces arrangements ou accords internationaux ne sont pas toujours faits pour être appliqués...

En réalité l'optimisme de la "bonne gouvernance", surtout à l'échelle internationale, repose fondamentalement sur une hypothèse centrale ; le passage d'un gouvernement par les Etats à un gouvernement par la société civile "mondiale", les scientifiques, les ONG, les entreprises, les institutions non majoritaires, les réseaux de collectivités locales (la société internationale). Comme le suggèrent Peter et Ernst $\mathrm{Haas}^{20}$, la matrice des régulations internationales ne se situe plus en effet dans la bonne volonté des Etats souverains, mais dans l'interaction complexe entre la connaissance scientifique, l'information, la stratégie des organisations internationales, les forces de marché, le jeu des groupes de pression, le pouvoir des villes et des régions,... C'est à partir de ces acteurs décentralisés que se crée, par contagion successive, par intégration culturelle, par apprentissage, la conscience commune des interdépendances globales. Et c'est parce que cette conscience commune se développe que la prise en compte des problèmes d'environnement peut se suffire de signaux faibles contenus dans des principes d'action généraux (comme "le développement durable"), ou dans des accords internationaux sans sanctions très claires.

L'important dans les "régimes internationaux" c'est donc moins en définitive ce qu'ils contiennent que les processus qu'ils ouvrent; parce que ceux ci pourront être appropriés par une société internationale résolument activiste et convaincue des avantages éthiques ou économiques - d'une action mutuelle. 

Mais faut-il, pour autant, surestimer son influence et sous estimer, inversement, celle des Etats ou des intérêts économiques? C'est la question que se posent ceux pour qui la "bonne gouvernance" est au moins autant, aujourd'hui, le problème que la solution face aux risques écologiques locaux ou globaux.

2. Le pessimisme de l'ingouvernabilité ou la gouvernance comme problème "gouvernabilité"; et le scepticisme des "réalistes", enclins à n'y voir qu'une illusion creuse ou naïve ; "un slogan permettant de dissimuler la réalité des conflits ou des jeux de pouvoir"22 ; une "trouvaille ne servant qu'à masquer l'impuissance de l'action publique ».

Ce scepticisme ne s'appuie pas seulement sur des arguments théoriques - parfois d'ailleurs contradictoires. Il s'alimente aussi de la relative faiblesse des résultats obtenus, jusqu'à présent, dans la mise en œuvre de ces nouvelles formes de gouvernance.

Il convient néanmoins de bien faire la distinction entre deux positions critiques de nature très différentes. Pour les uns la faiblesse des résultats constatés est essentiellement transitoire ou "locale": elle tient aux conditions dans lesquelles la participation démocratique est effectivement organisée - ou au temps d'acculturation nécessaire pour la mettre en place. Pour d'autres, au contraire, il y a contradiction structurelle entre la gouvernance démocratique et l'efficacité du "gouvernement"23 - certains allant même, à l'extrême, jusqu'à défendre une "incompatibilité radicale" entre démocratie d'opinion et prise en compte à long terme de l'environnement, comme a pu le faire, par exemple, Hans JONAS $^{24}$.

2.1. Un échec relatif des nouvelles formes de gouvernance?

50 Il est probablement prématuré de dresser, aujourd'hui, un bilan des nouvelles formes de gouvernance environnementale mises en place dans les années 90 : on manque, à l'évidence, du recul et des évaluations nécessaires; et il est, par définition, difficile de juger de l'efficacité de dispositifs dont l'objet est, d'abord,... d'ouvrir des processus.

51 Cette remarque étant faite, il faut bien malheureusement constater que dans beaucoup de situations où ces nouvelles formes de gouvernance ont été expérimentées ou proposées, les résultats ont été, jusqu'à présent, plutôt décevants - malgré l'indiscutable volonté de mobiliser la société civile.

On pourrait citer de multiples exemples: les tergiversations interminables sur l'effet de serre, et le retard - peut-être irréversible - pris dans la prévention de ce risque; l'incapacité à faire appliquer les conventions internationales; les progrès limités des "Agendas 21 " ou des politiques contractuelles au niveau local; les résultats encore incertains des "engagements volontaires" pris par les entreprises; la lenteur des politiques d'intégration ou d'incitation économique; les difficultés à étendre le débat public aux décisions réellement majeures: politiques, plans et programmes à moyen terme, ou grands choix technologiques... sans oublier le scepticisme assez général sur le "développement durable".

53 Assez significatif de cette tendance est le relatif échec du cinquième programme d'action européen, mis en place pour la période 1994-2000, et qui se caractérisait par une forte volonté d'innovation en matière de gouvernance. Le bilan publié récemment fait en effet ressortir une mise en œuvre tout à fait insuffisante de ce programme ; la progression trop lente de l'intégration de l'environnement dans les politiques sectorielles, l'incapacité à décider les instruments économiques envisagés au niveau communautaire, le manque de 
dispositifs de contrôle et d'évaluation, l'absence d'anticipation des nouveaux enjeux... Ajoutés à la confusion sur les OGM, la sécurité maritime, ou alimentaire, ou encore aux conflits avec l'Organisation Mondiale du Commerce, ces nombreux échecs témoignent de la difficulté pour les politiques de l'environnement de se passer d'objectifs et surtout de "mandats d'autorité" clairs. Il est néanmoins difficile de juger si toutes ces défaillances sont imputables à une gouvernance mal conçue... ou au contraire à un déficit de gouvernance.

2.2. Trois dérives : captation du débat public, impuissance et confusion des responsabilités, schizophrénie. Sans doute y a-t-il beaucoup de bonnes raisons pour imputer ces nombreux échecs aux contradictions de fond - politiques ou économiques - auxquelles se heurte la prise en compte de l'environnement (tableau 6). Mais pour les observateurs sceptiques, ces déceptions sont aussi la conséquence de trois dérives propres aux processus de gouvernance eux-même: la captation fréquente du débat public par une minorité de groupes d'intérêt ou d'experts; une attention trop faible à l'effectivité des politiques mises en place - ou une croyance illusoire dans des engagements trop flexibles; et enfin le constat d'un certain "découplage" entre politiques traditionnelles et politiques délibératives - qui conduit finalement à maintenir celles-ci dans une position marginale.

- La première critique sur la captation du débat public peut sembler paradoxale si l'on considère, par exemple, la progression considérable du nombre des ONG environnementales à l'échelle internationale ou locale. Il semble néanmoins que les efforts incontestables pour ouvrir les processus de la décision à la société civile ont jusqu'à présent surtout bénéficié à un nombre relativement limité de groupes d'intérêt bien organisés : représentants des grands lobbies industriels ou corporatistes (chasseurs... ), propriétaires riverains, experts scientifiques reconnus, grandes ONG internationales, représentants des administrations... Marie-Claude SMOUTS constate ainsi que dans le domaine des négociations internationales, "les populations indigènes, les communautés locales, les organisations villageoises - qui ont pourtant un rôle déterminant dans la mise en cuvre des solutions sur le terrain - sont restées peu représentées ${ }^{25}$ ". On pourrait en dire autant, en matière de gestion des risques, des populations les plus exposées; ou, dans le domaine de l'aménagement urbain, des habitants des quartiers défavorisés... 


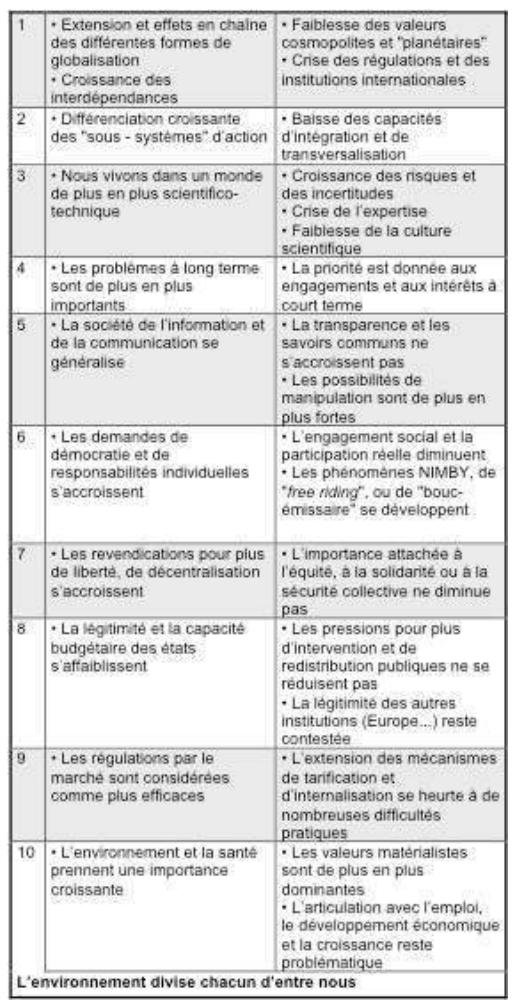

57 Cette éviction de certains des groupes les plus concernés ne traduit pas seulement la volonté évidente des institutions de "cadrer" et maitriser les processus de participation. Elle reflète également une des contradictions fondamentales de l'environnement - tout à la fois support virtuel d'un élargissement de la démocratie et lieu privilégié d'une certaine dépolitisation, au profit des experts. Car cette contradiction n'oppose pas seulement deux conceptions différentes de l'environnement, celle du public ou des écologistes contre celle des techniciens : elle traverse le mouvement associatif lui-même, à la fois défenseur et porte-parole de la démocratie contre les experts, et lui-même promoteur d'une "nouvelle classe" de spécialistes capables d'intervenir dans des débats de plus en plus techniques. Il y a là, naturellement un autre risque de captation du débat public : que la fonction de porte-parole du mouvement associatif soit absorbée par celle... de producteur d'expertises.

58 Au-delà de ce risque de captation du débat public, toute une littérature, comme on le sait extrêmement abondante, s'est, depuis plus de vingt ans, attachée à mettre en évidence toutes les limites - mais aussi les opportunités - d'une participation plus ouverte dans le champ de l'environnement. Les analyses sont en revanche, plus discrètes sur une seconde critique des modes de gouvernance qui porte, elle, sur la relation ou plutôt l'absence de relation entre ces processus de participation et la partie plus traditionnelle des politiques publiques - élaboration des lois, politiques réglementaires, décisions fiscales ou budgétaires, mise en œuvre du droit... autrement dit sur les relations entre "gouvernement" et "gouvernance".

Certains soupçonnent cependant le discours généreux sur la transparence démocratique de n'être qu'un paravent derrière lequel - ou à coté duquel - se restructurent les politiques traditionnelles. D'un coté est entretenue une très large confusion entre 
processus participatif et démocratie représentative - comme si les objectifs et les attentes pouvaient être de même nature ${ }^{26}$. De l'autre on constate un fréquent découplage entre les discours et les réalités - entre les consultations et les décisions ("the decide - announce defend - syndrom"), entre les décisions et leur mise en œuvre, entre les procédures formelles et les négociations informelles ( the front and the back office), entre les expériences de délibération et la démocratie représentative - comme s'il y avait de plus en plus de difficultés à articuler la politique de l'opinion et les grands choix stratégiques sur les problèmes ${ }^{27}$; d'où l'impression d'une certaine "schizophrénie de l'action publique", mais aussi le sentiment que les formes participatives ou délibératives de gouvernance sont le plus souvent cantonnées soit à une fonction d'alibi, soit à un rôle symbolique, soit à la gestion des crises - lorsque "les jeux sont faits", les décisions marginales, ou les pouvoirs publics fortement délégitimés.

Cela ne veut naturellement pas dire que le poids de l'opinion doive être en définitive relativisé : mais comme le montre très bien Christian Hey à propos de l'intégration de l'environnement ${ }^{28}$, une pression, même insistante, de l'opinion, si elle n'est pas relayée par la machine réglementaire, risque fort finalement de ne conduire qu'à des politiques purement symboliques - effets d'annonce sans lendemain, principes trop généraux ou décisions inapplicables...

61 Sans doute l'environnement a-t-il été un terrain particulièrement propice pour de telles " politiques virtuelles". C'est néanmoins surtout sous un troisième angle, celui de l'efficacité, que sont fondamentalement critiquées le nouvelles formes de gouvernance. Au vu des résultats acquis, ce soupçon d'inefficacité n'est pas, comme on l'a vu, infondé. Mais pour les plus sceptiques, cette impuissance serait en fait structurelle : comme Robert Dahl en fait l'hypothèse, il y aurait bien une contradiction de fond entre gouvernabilité et gouvernance, et peut-être même entre gouvernabilité et participation.

La crainte est qu'un usage excessif des consultations informelles, des contrats négociés, des réglementations flexibles, des incitations souples, des accords volontaires, des engagements révisables (soft consultations, soft laws, soft incentives, soft agreements, soft contracts, soft committments...) - sans objectifs clairs, sans mandats d'autorité pour les appliquer, ou sans sanctions ou moyens de contrôles efficaces - ce que Jean Leca a appelé "l'Etat creux"29, ne conduise finalement à des situations où aucune véritable décision n'est prise ou effectivement mise en œuvre. De manière encore plus immédiate, on peut légitimement se demander - surtout dans un pays où les structures territoriales sont aussi opaques qu'en France - si la dilution et la confusion des responsabilités qu'entraîne nécessairement la multiplication des partenariats et des formes souples de contrats n'est pas, à terme, complètement incompatible avec les exigences sociales croissantes de transparence et d'imputation des risques ${ }^{30}$. Tous les arguments mobilisés pour promouvoir la "bonne gouvernance" - la crédibilité, la responsabilité, "l'accountability", l'efficacité, sont ainsi "renversés" pour en faire la critique $^{31}$. Et se trouve tout particulièrement mise en doute sa capacité à promouvoir des bifurcations importantes ou d'aller au-delà de la "justice par avantage mutuel" (situation « win-win»).

Dans le domaine de l'environnement et de la gestion des risques cet argument de l'impuissance a une résonance très forte - non seulement à cause de la faiblesse structurelle des systèmes de contrôle et de sanction- notamment à l'échelle internationale - mais surtout en raison de l'importance du temps dans la mesure des résultats: compte tenu des phénomènes d'irréversibilité et des risques potentiels de catastrophes, il ne suffit pas de choisir une solution acceptable et efficace; il faut aussi 
qu'elle soit mise en œuvre au bon moment. C'est peut être ce risque d'inanité, d'inutilité de décision trop tardives, qui constitue la critique la plus forte et la plus radicale contre une certaine forme trop laxiste de la gouvernance : l'exemple de l'effet de serre montre en effet qu'il est extrêmement difficile de concilier un processus ouvert d'ajustement mutuel (decision making through mutual adjustement) ${ }^{32}$ et des phénomènes naturels, qui ont leur propre temporalité. Et la même remarque pourrait s'appliquer à tous les risques majeurs - du risque chimique comme à Toulouse au risque nucléaire ou aux grandes inondations. Toute la question est naturellement de savoir s'il y a bien, en l'occurrence, d'autres choix possibles.

2.3. Trop ou trop peu de gouvernance ? Un pessimisme à quatre faces

Il n'y a pas que sur le thème de l'effet de serre que la gouvernance démocratique suscite des débats et des critiques. Dans tous les domaines de l'environnement, celles-ci sont particulièrement nombreuses - sans doute d'abord en raison même des espoirs importants placés dans la participation, mais aussi parce que les questions posées à la démocratie par l'environnement n'ont pas de réponse simple :

- Comment organiser la confrontation des opinions sur des questions essentiellement médiatisées par la science, mais auxquelles celle-ci est incapable d'apporter des réponses claires?

- . Où débattre de problèmes d'environnement "transcendant" les frontières ou qui affectent des territoires qui n'ont rien à voir avec ceux des institutions traditionnelles?

- Comment rendre compatible le cycle court des politiques publiques avec la prise en compte des générations futures?

- Comment, enfin, introduire plus de justice et de transparence dès lors qu'il s'agit de distribuer des risques et non des avantages économiques ou sociaux? réalité sur quatre catégories d'argumentation extrêmement distinctes, que l'on a souvent tendance à confondre bien qu'elles puissent conduire à des conclusions radicalement différentes (tableau 7).

Tableau 7 : Quatre approches critiques de la gouvernance

\begin{tabular}{|c|c|c|}
\hline & $\begin{array}{l}\text { Critique des movens } \\
\text { (procédural) }\end{array}$ & $\begin{array}{l}\text { Critique des } \\
\text { objectifs } \\
\text { (substantialiste) }\end{array}$ \\
\hline $\begin{array}{l}\text { Pessimisme } \\
\text { reformatiste } \\
\text { (Inadaptation ou } \\
\text { transition) }\end{array}$ & $\begin{array}{l}\text { Dysfonctionnement ou } \\
\text { limitation des } \\
\text { processus de } \\
\text { participation existants. }\end{array}$ & $\begin{array}{l}\text { Inefficacite de } \\
\text { politiques dépourves } \\
\text { de mandats d'autorite } \\
\text { Limite de TEtat } \\
\text { creux. }\end{array}$ \\
\hline $\begin{array}{l}\text { Pessimisme radical } \\
\text { (inadaptation } \\
\text { structureile) }\end{array}$ & $\begin{array}{l}\text { Culture de la } \\
\text { participation } \\
\text { insulfisante } \\
\text { Indifference } \\
\text { démocratique et repil } \\
\text { individual ste. }\end{array}$ & $\begin{array}{l}\text { Incapacite de la } \\
\text { democratie a prendre } \\
\text { en compte le long } \\
\text { terme et les risques. }\end{array}$ \\
\hline
\end{tabular}

67 Très schématiquement les critiques de la gouvernance se structurent en effet autour d'une double opposition :

- pour les uns ce sont les conditions de mise en œuvre qui sont critiquables ; pour d'autres ce sont, au contraire, les objectifs eux-mêmes ;

- ce qui est pour les uns une question de transition, est vu par les autres comme une inadaptation structurelle ; 
un premier groupe, largement majoritaire, d'observateurs critiques, ce sont essentiellement les conditions dans lesquelles les politiques délibératives sont mises en œuvre qui incitent au pessimisme : un accès au débat encore trop inégal, le pluralisme non respecté, une autonomie réduite des acteurs consultés, l'équivalence des intérêts mal garantie, une interaction entre parties prenantes beaucoup trop faible, des procédures marginalisées, des conclusions non publiées ou sans suite opératoire... Tout cela justifie un certain scepticisme, mais un scepticisme tempéré: on reste dans le registre des dysfonctionnements pour lesquels il existe, en principe, des solutions managériales ${ }^{33}$.

Dans une seconde perspective beaucoup plus pessimiste, ce ne sont pas les procédures qui sont contestées, mais plutôt l'existence des conditions culturelles nécessaires pour les faire fonctionner. L'hypothèse d'un activisme de la société civile, d'une mobilisation spontanée des acteurs est fortement questionnée. On lui oppose le constat - qui vaut surtout pour les problèmes globaux - d'une très large indifférence démocratique. Comme on l'a déjà évoqué, le point faible de tous les espoirs placés dans la gouvernance est en effet que ceux-ci reposent sur le présupposé de transformations culturelles importantes: l'existence d'acteurs intéressés à s'engager dans l'action collective, une sensibilité suffisante au problème posé, un minimum de connaissance et de confiance réciproque. Or l'expérience historique a clairement montré, qu'en dehors des problèmes locaux, cette transposition vers "l'autorégulation" réussie pouvait prendre souvent un temps très long : un siècle, par exemple, en France, pour passer à une prise en charge par les usagers de la qualité de l'eau. (voir les graphiques 1 et 2).

Certes la circulation de l'information n'a plus rien à voir aujourd'hui avec ce qu'elle était il y a un siècle : mais on sait aussi avec quelles difficultés se fait le passage entre l'opinion publique et la connaissance scientifique; et l'importance décisive du "knowledge gap", considéré par Robert Dahl comme le principal obstacle à la démocratie. Il y a donc clairement un risque que la participation arrive toujours avec un "train de retard" - ce qui rejoint le problème de l'inanité dont on a vu, précédemment, l'enjeu.

Dans une troisième perspective critique, les échecs de la gouvernance s'expliquent beaucoup plus simplement - par l'inadéquation de ses objectifs.

Ce qui est ici mis en cause, c'est une certaine dérive libérale et néo-corporative de la gouvernance qui tend à en faire un substitut des formes classiques d'action publique ; l'illusion d'une "pilotless policy" 34 , sans mandat d'autorité, sans hiérarchisation des intérêts, sans institutions de contrôle. L'idée, aussi, que la participation - dans un contexte de crise - pourrait efficacement remplacer les formes électives de la démocratie. Dans cette troisième perspective, qui donne la priorité aux structures de gouvernabilité, le risque majeur en matière d'environnement apparaît en effet moins celui d'un manque de légitimité des décisions publiques que celui d'un affaiblissement des institutions - en particulier aux niveaux international et local (voir encarts 1 et 2); d'une absence de leadership face à des rapports de force défavorables; d'une dilution des responsabilités liée, par exemple, à la multiplication des partenariats ou à une décentralisation mal maîtrisée ; et finalement d'une impuissance de la démocratie représentative face à un double phénomène de polarisation, autour des relations producteurs - consommateurs, et autour des phénomènes de "glocalisation" (convergence des réseaux locaux et supranationaux d'action publique) ${ }^{35}$. Ce n'est donc pas l'ambition d'une plus grande ouverture démocratique qui est critiquée, mais plutôt la tentation de faire de la gouvernance un nouveau mythe politique se substituant aux autres. 
73 A cela s'oppose une quatrième et dernière position, encore plus radicalement pessimiste, qui met, elle, directement en doute à travers la notion de gouvernance la capacité même des démocraties à prendre en compte leurs responsabilités à long terme - en particulier dans le domaine de l'environnement. S'appuyant sur Hans Jonas et son "Principe responsabilité", certains mouvements écologistes opposent en effet à "l'indifférence démocratique" la nécessité d'une prise en charge par une élite éclairée de l'avenir à long terme de la planète. Exprimé de cette manière, l'argument est difficilement recevable. Mais dans un registre plus modéré, certains experts reconnus, comme Giandomenico Majone, plaident pour le développement d'institutions non majoritaires ${ }^{36}$ ou d'autorités indépendantes, capables de garantir le respect d'objectif à long terme en s'affranchissant des incertitudes de la démocratie élective. L'explosion, en Europe, de la création de ces autorités montre bien, là encore, qu'il ne s'agit pas, malgré les apparences, d'une critique abstraite.

74 Comme on le constate à la diversité des arguments précédents, les perspectives ouvertes par la gouvernance environnementale suscitent donc beaucoup de controverses et pas mal d'interrogations. Cette perplexité traduit sans doute la difficulté d'articuler démocratie et complexité, démocratie et expertise. Mais elle témoigne aussi de la vivacité $\mathrm{du}$ débat qui, autour du thème de l'environnement, oppose différentes conceptions alternatives de la démocratie.

\section{Encart 1 Des régulations et des institutions internationales affaiblies}

Dans l'hypothèse qui est celle des "régimes internationaux", la coordination des actions décentralisées pourrait très bien, en principe, se contenter de "signaux faibles" et d'une autorité internationale limitée.

Certains pensent, au contraire, que l'un des problèmes majeurs de la gouvernance actuelle, dans un contexte de globalisation, est la faiblesse croissante des institutions internationales. Dans le domaine de l'environnement global, Magnus ANDERSON et Joyeeta GUPTA ont bien mis en évidence l'importance de ces faiblesses, en en faisant un inventaire très précis $^{37}$ :

- . "beaucoup des institutions existantes ont des mandats étroits, des budgets réduits et une légitimité contestée" ;

- . "les mécanismes de coordination entre institutions sont insuffisants et il n'y a pas de leadership clairement affiché" ;

- . "la politique des institutions reflète les relations de pouvoir existantes au sein des Nations Unies - au détriment de l'environnement et de son intégration dans les autres politiques" ;

- . "les institutions manquent d'indépendance à cause de leur vulnérabilité financière" ;

- . "l'aide internationale n'a pas atteint les niveaux attendus" ;

- . et enfin, "les moyens, mis à disposition des institutions internationales pour évaluer la mise en œuvre des accords signés, restent notoirement insuffisants".

Beaucoup de réformes ont été récemment proposées pour remédier à ces défaillances, et faire face à cette crise. Mais il faut bien constater que la majorité des ces tentatives se sont soldées par un échec. Sans évoquer l'hypothèse d'un gouvernement mondial, il a, par exemple, été suggéré de créer un second Conseil de sécurité, de transformer le PNUE en une "Agence mondiale du développement durable", de mettre en place une "Cour Internationale d'arbitrage des conflits liés à l'environnement", de créer une "Autorité mondiale des climats", ou de refondre le système des Nations Unies. 
Aucune de ces réformes n'a abouti.

Encart 2 : Des institutions locales inadaptées

En France, ces conditions ne sont pas réunies. Malgré les réformes récentes, la «transition institutionnelle » vers une décentralisation équilibrée reste inachevée; et il subsiste de très nombreux handicaps à une gestion efficace et équitable de l'environnement au niveau local. Ces handicaps sont connus : émiettement communal et manque d'autonomie de la majorité des petites communes; multiplication incohérente et absence de coordination des différents niveaux de pouvoirs; faible légitimité démocratique des structures intercommunales; déséquilibre des pouvoirs de police au profit des polices d'Etat spécialisées - soufrant elles-mêmes d'un manque chronique de moyens ; dépendance des collectivités locales par rapport aux sociétés concessionnaires ; cloisonnement durable des services déconcentrés de l'Etat et déficit de réflexion sur la territorialisation des politiques publiques; faiblesse relative des régions et des pouvoirs d'agglomération; déficit chronique des procédures de participation du public; manque d'instruments d'évaluation et d'information...

81 Dans un pays marqué par de très grandes inégalités territoriales, ces défaillances institutionnelles ont comme traduction concrète des différences de plus de un à cent dans les expositions aux risques ou l'accès à la nature.

3. Vers une « démocratie cognitive »

82 Opposer l'optimisme de la "bonne gouvernance" au réalisme de l'action - ou au pessimisme radical de "l'ingouvernabilité" - est stérile. Ce qu'il faut, au contraire, c'est essayer de construire des passerelles entre ces deux univers qui ont souvent tendance à s'ignorer. Tirer profit des opportunités d'innovation incontestablement ouvertes par la gouvernance participative sans tomber dans l'incantation, la manipulation déguisée, ou la démagogie hypocrite.

83 Les réflexions sur la gouvernance, en opérant le déplacement d'une conception classique du gouvernement, centrée sur l'Etat, à une conception beaucoup plus ouverte de l'action collective, centrée sur une pluralité d'acteurs exprimant une multiplicité d'intérêts, correspondent le mieux aux réalités du monde actuel, à la crise de légitimation de l'autorité publique, et aux exigences de la démocratie. Mais la gouvernance n'est, comme on l'a vu, qu'un ensemble de dispositifs, de procédures, d'instruments qui ne peuvent fonctionner que s'ils sont appropriés, mobilisés par des acteurs ayant un accès équitable à la décision, partageant un minimum d'intérêt commun, et disposant de marges de manœuvre suffisantes. Son efficacité, sa crédibilité, sont donc liées à des conditions bien précises.

Dans le cas de la gestion de l'environnement, et en particulier pour les risques globaux, la situation de départ est celle de problématiques mal structurées, d'acteurs nombreux et mal identifiés, d'intérêts fortement divergents, et de rapports de force défavorables. Les 
conditions d'une "bonne gouvernance" ne sont pas réunies à priori : elles doivent donc être en partie construites.

85 Cette construction semble devoir s'articuler autour de quatre grands axes, qui ne seront qu'esquissés ici pour conclure :

- - rendre les procédures de gouvernance plus crédibles (condition méthodologique);

- - rééquilibrer les rapports de force (condition politique) ;

- - redéfinir les lignes de partage entre "gouvernance participative" et "gouvernement représentatif" (condition institutionnelle);

- - et enfin, promouvoir une "démocratie cognitive" (condition culturelle).

3.1. Rendre les processus de gouvernance plus crédibles

86 A l'évidence on est encore, en matière d'environnement dans une phase d'adolescence des processus de gouvernance - ce qui veut dire que d'importantes étapes restent encore à franchir pour leur assurer une crédibilité suffisante. En réalité, cette situation n'est pas étonnante. Comme le remarque Giandoménico MAJONE, "Il aura fallu dans les domaines traditionnels de la délibération publique plusieurs siècles pour que les règles du débat soient transformées en institutions durables, et la complexité inhérente aux problèmes d'environnement rend cette institutionnalisation dans les nouveaux espaces du débat encore plus difficile, ne serait ce qu'en raison de leurs dimensions transnationales ${ }^{38}$."

En aval de la décision, beaucoup reste à faire, comme on l'a dit et répété, pour améliorer les conditions dans lesquelles la participation et le débat public sont organisés : multiplier les opportunités de consultation; en diversifier les formes ; mieux les lier à la décision ; cadrer les procédures; les rendre plus autonomes et plus interactives ; favoriser une expertise pluraliste... On sait très bien, par exemple qu'à l'échelle européenne les conditions dans lesquelles l'expertise est convoquée et menée ne sont généralement pas des plus satisfaisantes : l'urgence, la contingence, les contraintes extérieures, la brièveté des sessions, le poids des lobbies, la confusion institutionnelle, les problèmes de culture... ne permettent pas toujours, un débat ouvert et serein. Tout cela, en principe appelle des solutions pragmatiques, dans un registre qui est celui du "design institutionnel" : mais, comme au niveau national, il est probable que cela suppose d'abord un accord constitutif sur la nécessité de nouvelles règles de jeu.

En amont de la décision, l'efficacité du débat suppose aussi un réinvestissement considérable dans l'évaluation - évaluation des politiques publiques, études d'impacts de plans et programmes, évaluations technologiques, méthodes de suivi et de contrôle... Globalement on peut affirmer que la pauvreté des informations et des connaissances dont on dispose en matière d'évaluation est en effet un des obstacles majeurs à une mobilisation structurée en matière d'environnement. On reste très loin $\mathrm{du}$ cercle vertueux de la "société réflexive" - transparente à elle-même. Ce souci de l'évaluation devrait d'ailleurs s'accompagner d'une volonté plus soutenue de simplification des institutions et des procédures - sans laquelle la confusion des responsabilités restera la règle générale, et la transparence l'exception.

Enfin un effort méthodologique reste incontestablement à faire pour redonner à la gestion du temps le rôle central qu'elle devrait avoir dans les processus de gouvernance. Cela ne renvoie pas seulement aux méthodes de prospective - ou à la prise en compte des générations futures. Mais à la nécessité de confronter en permanence la virtualité des promesses et la réalité des actions mises en œuvre. Réussir à intégrer les contraintes de temps dans le débat public - comme cherchent à le faire le principe de précaution ou le 
développement durable - serait naturellement, pour l'environnement, un progrès majeur.

3.2. Construire des rapports de force plus équilibrés

Tous les efforts en matière de "design institutionnel" risquent cependant de s'avérer insuffisant s'ils ne s'accompagnent pas, parallèlement, d'un certain nombre d'actions visant à mieux équilibrer les rapports de force entre parties prenantes.

Cela passe sans doute par la concrétisation d'une ou plusieurs des propositions suivantes :

- - une organisation plus formelle et plus cohérente des réseaux d'acteurs potentiellement intéressés par une prévention active des risques environnementaux et notamment des risques majeurs ou globaux ("de nouvelles alliances") ;

- - une "discrimination positive" au profit des groupes traditionnellement exclus du débat public et des producteurs de "contre expertise" ;

- - la relance du débat sur la constitution d'une autorité mondiale de l'environnement, ainsi que sur la réforme du système institutionnel international (de l'OMC aux agences des Nations Unies) ;

- - Un rééquilibrage significatif des compétences en matière d'environnement au profit des régions et des pouvoirs d'agglomération, s'accompagnant d'une véritable réflexion sur la notion de « subsidiarité »;

- • un décloisonnement des institutions chargées de l'environnement au sein de la Commission Européenne ou des « gouvernements » nationaux et régionaux, et plus généralement le développement de structures assurant la transversalité et l'intégration des intérêts ;

- - un leadership plus affirmé de l'Europe sur les questions de l'environnement global - ce qui suppose également de revoir les conditions d'harmonisation ou d'élaboration des politiques européennes ;

- - la mise en place de formes originales de financement permettant de réorienter les capitaux vers les besoins urgents des pays du sud, ou, en France, vers les territoires marginalisés ;

- et enfin la création de nouvelles agences d'expertise et de contrôle ayant une autonomie d'action et des pouvoirs de sanction réels.

Sans ces transformations il est à craindre que la mobilisation de la société civile ne fasse en effet que renforcer les évolutions déjà engagées.

3.3. Redéfinir les lignes de partage entre "gouvernance participative" et gouvernement représentatif

Au-delà de ces rapports de force, la question est également posée d'un meilleur équilibre entre "gouvernance participative" et "gouvernement représentatif", autorégulation de la société et autorité ou responsabilité des institutions.

La prise en compte de l'environnement ne peut tirer que des avantages d'une implication et d'un pouvoir renforcé de la société civile - des villes, des régions, des entreprises, du grand public... Mais ni la démocratie, ni l'environnement futur n'auraient à gagner d'une situation où ce renforcement se ferait contre les formes classiques de représentation élective ou au prix d'un affaiblissement des institutions publiques. Il ne s'agit pas, en d'autres termes, de remplacer le "gouvernement" par la "gouvernance", mais plutôt d'organiser leur articulation. On ne peut se contenter de constater la juxtaposition de logiques et de modes de régulation différents, se développant à plusieurs échelles - régulation de l'Etat, régulation du marché, régulation de la société civile... - il faut aussi penser leur combinaison - ce qui veut dire, sans doute, dessiner de nouvelles lignes de partage. 

de partage, entre démocratie participative et démocratie représentative (parlement et gouvernement) pourrait s'organiser autour des différentes étapes du cycle de vie politique - mise sur agenda, décision, mise en œuvre, évaluation et contrôle - la participation étant le plus souvent limitée à la phase de mise en œuvre et d'acceptation des projets. On sait aujourd'hui que cette division fonctionnelle est très largement dépassée.

Une autre ligne de partage, beaucoup mieux argumentée, conduit plutôt à séparer la résolution de problèmes concrets et la distribution de droits généraux - les questions d'efficacité et les questions de redistribution ${ }^{39}$.

Pour Patrice DURAN par exemple, "la participation ne vaut qu'en tant qu'instrument de résolution des problèmes de société, et repose clairement sur d'autres logiques que celles qui animent la démocratie politique. Ce ne sont pas, dans ce cas, les jeunes, les vieux, les femmes, dont il faut assurer la représentativité; ce sont les problèmes qu'il faut gérer avec ceux qui les vivent - ce qui n'est pas la même chose... ${ }^{40 "}$ On est donc face à deux logiques d'action bien distinctes, dans une configuration très proche de la "subsidiarité active", avec deux modes d'implication démocratique sensiblement différents - mais qu'il s'agit d'articuler.

Pour l'environnement, il semble en effet qu'une vision dualiste de l'organisation des pouvoirs, combinant l'unité et la diversité, la légitimité et l'efficacité, la mobilisation et " l'accountability", soit la mieux appropriée.

Aux Etats, aux parlements, aux juges de prendre en charge, dans une perspective de solidarité et de protection à long terme, les risques écologiquement ou socialement intolérables, d'assurer "un filet de sécurité" contre les accidents majeurs, de garantir (par l'incitation ou la sanction) l'application effective des règles - en anticipant éventuellement la demande sociale. Aux collectivités locales, aux entreprises, à la société civile de négocier et gérer collectivement les problèmes qui les concernent, en construisant des visions partagées et en intervenant dans des procédures ouvertes de codécision.

Raisonnable et sans doute réaliste, cette nouvelle division du travail - qui conduit à recentrer l'Etat sur les situations insoutenables, et les procédures de participation sur la résolution de problèmes (problem solving) - laisse cependant mal résolue deux questions majeures. D'abord celle de l'intervention du citoyen dans le contrôle de la techno-science. Et ensuite, et peut être surtout, celle de la participation du public et des différentes parties prenantes dans la gestion de problèmes non locaux - notamment à l'échelle européenne ou mondiale. Pour l'instant les seules réponses crédibles à ces deux défis semblent bien être le développement et l'intervention croissante de structures de médiation et de transversalisation, situées à l'interface entre démocratie directe et démocratie représentative: régions, autorités indépendantes, forums de citoyens, conférences des consensus, fédérations associatives... . Mais on voit bien qu'il s'agit là de solutions transitoires, en attendant des recompositions institutionnelles qui devront être beaucoup plus profondes... ${ }^{41}$

Promouvoir une "démocratie cognitive"

101 La promesse d'un accès plus ouvert de la société civile à la décision risque malheureusement de rester purement formelle, si elle ne s'accompagne pas, 
parallèlement, de profonds changements culturels, et surtout d'une "nouvelle donne" (" $a$ new deal") dans le partage du savoir et de l'expertise.

La "bonne gouvernance" accorde, comme on l'a vu, une place centrale à l'information et à la connaissance, non seulement comme condition de mise en œuvre des politiques normatives, mais aussi comme alternative possible à ces politiques. Il est clair cependant que l'information ne suffit pas pour construire une culture commune, ni pour produire de la mobilisation collective: il faut aussi pouvoir la relier à l'action quotidienne des individus, à leurs possibilités concrètes d'intervention dans la vie démocratique, et, plus largement, à une mémoire partagée.

- - Le premier défi à relever, notamment dans le cas des problèmes globaux, est de faire émerger une culture commune de la globalité, une opinion publique mondiale. Paradoxalement beaucoup pensent que cette conscience planétaire n'a de chance de naitre que si elle s'enracine dans la réalité des communautés locales - à condition que celles-ci puissent s'engager sur des projets précis. Mais beaucoup reste à faire pour que le "penser globalement - agir localement" devienne autre chose qu'un slogan sans portée.

- - Le second défi, encore plus redoutable, est de réduire le fossé, qui ne cesse de grandir, entre l'expert et le citoyen ("the knowledge gap") - ce qui renvoie, pour l'environnement comme dans d'autres domaines, au rôle fondamental de l'éducation, à l'émergence d'une véritable culture du risque et surtout à la construction de ce qu'Edgar MORIN a appelé une "démocratie cognitive".

Comment imaginer cette démocratie cognitive, ce nouveau contrat social entre science, société et politique? On peut en définir, pour conclure quelques unes des conditions :

- - du coté des médias un effort persévérant de pédagogie et de distanciation par rapport à l'événement;

- - du coté des scientifiques, une éthique de l'objectivation et de l'autonomie qui mette le savoir à l'abri de toute forme d'expropriation par les groupes de pression; et un souci réel de mise en débat public des connaissances ;

- - du coté du système éducatif, la généralisation à tous de l'enseignement scientifique, et une meilleure intégration de l'environnement dans les filières de formation;

- - du coté des industriels, l'acceptation du principe de précaution et de la transparence ;

- . du coté de l'expert, une plus grande ouverture aux aspects sociaux et éthiques des problèmes dont il a la charge, et le souci, non pas de fournir des solutions toutes faites mais de rendre possible "la négociation sur les valeurs qu'institue le thème de l'environnement" (F. EWALD) ;

- - du coté des autorités publiques, la transparence des objectifs et des informations et surtout l'acceptation de la pluralité de l'expertise comme instrument majeur de la démocratie - ce qui implique la volonté tenace de réduire partout les obstacles qui s'opposent au développement de la contre expertise ;

- - du coté des institutions, la mise en place de règles permettant d'éviter la confusion des pouvoirs, et la création de procédures ou de structures de médiation permettant d'assurer un véritable contrôle démocratique des choix scientifiques et techniques (rôle des autorités indépendantes);

- . et enfin du coté de l'opinion, une acculturation au doute et à l'idée que la connaissance ne se confond pas avec l'accumulation de certitudes toutes faites - ce qui suppose aussi une certaine acceptation du droit à l'erreur pour la politique. 
104 on le constate finalement à travers toutes ces préconisations, la «bonne gouvernance " suppose donc des changements structurels et un ensemble d'innovations qui vont manifestement très au-delà du bricolage pragmatique et des " arrangements de terrain » dans lesquels on la cantonne généralement. Cette remarque ne semble pas devoir être limitée au seul cas de l'environnement. Elle devrait sans trop de difficultés pouvoir s'appliquer à la plupart des domaines de l'action publique, et notamment - c'est l'hypothèse que l'on fera ici pour conclure - à l'aménagement du territoire.

\section{NOTES}

1. Sur ces critiques, voir, en particulier : J. PIERRE (Ed) «Debating governance », Oxford University Press, 2000 ; Patrick LE GALES, « Government and governance in European cities - Implication for democracy », papier non publié présenté à la conférence de Florence de septembre 2000 ; ainsi que Jean-Gustave PADIOLEAU : « Les gogos de la gouvernance »-Libération, $1^{\text {er juin } 2000 .}$

2. Source: W. D. SUNDERLIN, " Global environmental change, sociology and paradigm isolation ". Global environmental change, Vol. 5, Number 3, June 1995

3. Voir, sur cet aspect théorique, le papier préparé pour le séminaire de Florence sur la

“Gouvernance participative " par Jan KOOIMAN : "Governance, a socio-political perspective", in Jürgen R. GROTE et Bernard GBIKPI (Eds), « Participatory governance », LESKE-BUDDRICH, 2002

4. Dans ce même document, Jan KOOIMAN cite une dizaine de définitions différentes de la "gouvernance".

5. Source : Webster's New Dictionnary ; London, Dorset and Barber, 1979.

6. Source Christophe BAIL, "Environmental Governance : Reducing risks in democratic societies". Introduction paper, EEC, Future Studies Unit, 1996.

7. Source : Pierre CALAME, «Des procédures de gouvernement au processus de gouvernance ». Séminaire d'ODENSE, 11 octobre 1996, Commission Economique européenne.

8. Olivier GODARD, « Stratégies industrielles et convention d'environnement. De I'univers stabilisé aux univers controversés », INSEE méthode 1993, Paris.

9. Source: G. VAN VLIET et Carlos MATUS. «Planification en sistemas de baja governabilidad », IDRI, Bogota, 1982.

10. Yves MENY et Jean-Claude THOENIG . Politiques Publiques ; Presses Universitaires de France, Paris, 1989.

11. Il faut rappeler que le mot « cybernétique » signifie au sens propre : "action de manœuvrer un vaisseau" ou, au sens figuré : "action de diriger, de gouverner".

12. Karl DEUTSCH, The Nerves of Government, New York, Free Press, 1963.

13. Source : Michel FOUCAULT, De la Gouvernementalité ; cours donné au collège de France en 1978 et 1979, Edition du Seuil 1989, Paris. 
14. Rappelons que dans ce modèle libéral, "il s'agit d'assurer la prospérité et l'autonomie des individus tout en les garantissant contre les insécurités majeures : le problème est essentiellement de « gouverner à la limite du trop et du trop peu de gouvernement » ou de définir le minimum de sécurité compatible avec la protection contre les risques inacceptables".

15. On pense naturellement aux travaux de GIDDENS, SIMON ou HABERMAS.

16. Voir, en particulier la description faite par FUKUYAMA de "I'ordre du monde" dans un futur "post-historique" dominé par les démocraties libérales, et les critiques sévères qu'il porte sur "l'irréalité du réalisme" en politique internationale (F.FUKUYAMA, The End of History and The Last Man, Free Press, 1992).

17. Titre du livre écrit en 1975 par Pierre BIRNBAUM (Edition du seuil) dans lequel l'auteur fait, par anticipation, une critique très lucide de l'approche fonctionnaliste de la gouvernance.

18. On a effectivement assisté, à l'échelle internationale, à une très large intégration du discours économique dans les logiques d'argumentation utilisée par les organisations non gouvernementales.

19. Source : Jan van TATENHOVE, "Political modernization and environmental policy", in J. THEYS (Ed.) : The environment in the $21^{\text {st }}$ century, GERMES, VOLUME 1, 1998, colloque de Fontevraud.

20. Peter HAAS - "Environmental institutions and evolving international governance". working draft, Harvard University, 1995 (Communication également présentée au colloque de Fontevraud, 1996)

21. Source : Paul WAPNER "Politics beyond the State : Environmental activism and world civic politics" ; World Politics, Vol. 47, N³, 1995

22. Source : Nicolas TENZER. «De quelques paradoxes de l'action dans les démocraties modernes. » - in, Prospective pour une gouvernance démocratique, Editions de l'Aube, 2000.

23. C'est la position défendue, par exemple, par Robert DAHL, lorsqu'il oppose "participation des citoyens" et "gouvernabilité" (System Effectiveness)

24. Hans JONAS : Le principe responsabilité, Editions du CERF, Paris, 1990 (Publication originale en 1979)

25. Marie-Claude SMOUTS : "La coopération internationale : de la coexistence à la gouvernance mondiale" ; in Les Nouvelles Relations Internationales, Presses de Science Po, 1998

26. Voir sur cette confusion l'article de Patrice DURAN : «Action publique, Action politique », in Gouvernance, territoire et citoyenneté urbaine, Editions PEDONE, 2000.

27. Un bon exemple de ce découplage est la conférence de citoyens organisé en France en juin 1998, sur le thème des OGM : bien que tenue à l'initiative de l'Office parlementaire des choix technologiques, cette conférence n'a eu aucune retombée sur les débats ou initiatives des parlementaires, qui n'y ont pas assisté.

28. Christian HEY : "Pillarisation and Prévalence of National Souvenegnity as Barriers, for Greening Sectoral Policies", Conférence on democratic and participatory governance Florence, ouvrage cité.

29. Source Jean LECA, «I'Etat creux », in La France au-delà du siècle, Editions de l'Aube, DATAR, 1994 
30. Voir, sur ce thème, l'article publié par Julien DAMON dans le numéro de mars 2002 de Futuribles : «La dictature du partenariat : vers de nouveaux modes de management public ?».

31. Voir en particulier les critiques très argumentées, développées par Jean-Gustave PADIOLEAU, dans : "La société du risque, une chance pour la démocratie", Le Débat, GALLIMARD 2000.

32. Titre du livre écrit en 1965 par Charles LINBLOM

33. Tout un ensemble de travaux s'attache en effet à définir les "règles du jeu" d'une participation efficace. Voir par exemple Patrice DURAN, (opus cité), mais également Pierre LASCOUMES : «Information, consultation expérimentation ; les activités et les formes d'organisation au sein des forums hybrides », CNRS, 1997, programmes " Risques collectifs et situations de crise", Paris.

34. Expression proposée en 1958 par Norton LONG

35. Source : Jan VAN TANTENHOVE : "Political modernization and environmental policy", in Jacques THEYS : L'environnement au XXI siècle, Editions GERMES, 1998.

36. Giandomenico MAJONE : "Temporal consistency and policy credibility : Why democracies need non majoritarian institutions ?", in Jacques THEYS (ed) The environment in the $21^{\text {th }}$ century, GERMES, PARIS, 1998.

37. Source : M. ANDERSON et J. GUPTA : "Adressing the institutionnal gaps in Global Environmental Governance", Colloque de Fontevraud, 1996, in Jacques THEYS (ed) L'environnement au XXlème siècle, GERMES, Tome I, 1998.

38. source : G.MAJONE, « Décisions publiques et délibération », in Revue française de science politique, vol 44, n4, août 1994

39. Distinction également proposée par Giandoménico MAJONE (opus cité)

40. Patrice DURAN : «Action publique, Action politique », in Jean Philippe LERESCHE (ed) Gouvernance territoriale et citoyenneté urbaine : de la coordination à la légitimité, PEDONE, 2001.

41. Les propositions de Bruno LATOUR en France ou d'Ulrich BECK en Allemagne visant à créer une instance parlementaire spécifique ("Parlement des choses" ou "Chambre Haute") pour traiter des problèmes situés entre science, technique et société vont dans ce sens.

\section{RÉSUMÉS}

Le terme de "gouvernance" s'est imposé au cours des années 90 comme le symbole d'une nouvelle modernité dans les modes d'action publique et est devenu, en quelques années, un des lieux communs du vocabulaire de l'aménagement du territoire. Néanmoins, cette banalisation, comme les critiques qu'a pu susciter cette notion, ne doivent pas faire oublier les potentialités d'innovations qu'elle recèle. Partant de ces constats et de ce postulat, l'auteur propose dans cet article une réflexion théorique et critique sur la notion de "gouvernance » en s'appuyant notamment sur les innovations en la matière qui ont eu pour cadre le champ environnemental depuis une vingtaine d'années. La notion de gouvernance et les usages qui en sont fait sont 
interrogés directement dans un premier temps, afin d'en affiner le contenu au-delà de la simple définition de "boîte à outils" managériale. Les potentialités théoriques de la notion de gouvernance sont ensuite discutées et critiquées à partir des exemples concrets d'innovations procédurales qui se sont multipliées dans le champs de l'environnement.

INDEX

Keywords : gouvernance

Mots-clés : environnement, innovations procédurales, gouvernabilité, gouvernementalité, démocratie cognitive

\section{AUTEUR}

\section{JACQUES THEYS}

Directeur scientifique et directeur adjoint de l'Institut Français de l'Environnement (IFEN). Il est également Directeur du Centre de prospective et de veille scientifique (CPVS) à la Direction de la Recherche, des Affaires scientifiques et Techniques, Ministère de l'équipement, des logements et des transports. 